\title{
Effect of in vitro digestion on polyphenol release and antioxidant activity from three different species of dragon fruit (Hylocereus sp)
}

\author{
M. I. M. Flores-Verastegui, H. Lightowler and P. S. Thondre \\ Oxford Brookes Centre for Nutrition and Health, Department of Sport, Health Sciences and Social Work, Faculty of \\ Health and Life Sciences, Oxford Brookes University, Oxford, OX3 OBP, UK
}

The consumption of plants and derivatives rich in polyphenols is known to provide a positive effect on human health ${ }^{(1)}$. However, the phenolic compounds in a food matrix are not always completely accessible to digestive fluids. Therefore, in vitro digestion has been used as a mechanism to determine the bio-accessibility of polyphenols, and their possible health effects ${ }^{(2)}$.

Dragon fruit is a tropical fruit that has been considered as an alternative treatment for diabetic people in different regions of the world and some studies have determined their content of phenolic compounds ${ }^{(3,4)}$. However, their bio- accessibility from different forms of the fruit has not been reported. The aim of this research was to quantify the polyphenol release and their antioxidant capacity during in vitro digestion of three different species of dragon fruit.

An in vitro digestion was carried out for red flesh dragon fruit (Hylocereus polyrhizus), white flesh dragon fruit (Hylocereus undatus), and yellow peel dragon fruit (Hylocereus megalanthus), under fresh, frozen, and dried conditions. Samples were taken at baseline, gastric phase, as well as 20, 60, and 120 minutes during intestinal phase. The amount of total phenolic compounds released was determined by Folin-Ciocalteu method and the antioxidant activity was evaluated by DPPH (2, 2-diphenyl-1-picrylhydrazyl) radical scavenging analysis. Depending on the normality of the results determined by Shapiro-Wilk test, a one-Way ANOVA with a Tukey's post hoc analysis or Kruskal-Wallis non-parametric test was carried out. Statistical difference was set at $\mathrm{p}<0.05$.

The results showed a significant difference in the amount of polyphenols released between digestion phases $(\mathrm{p}<0.001)$ and an increase in their bio-accessibility as the in vitro digestion progressed. During intestinal phase, dried fruits presented lower release of total phenolic compounds and the white flesh dragon fruit exhibited the lowest value $(538.54 \pm 64.59 \mu \mathrm{g}$ of gallic acid equivalent (GAE)/g fresh weight (FW)). On the other hand, fresh and frozen fruits had higher release of polyphenols with red flesh dragon fruit presenting the highest levels $(2846.75 \pm 333.10 \mu \mathrm{g}$ GAE/g FW and $2737.18 \pm 126.87 \mu \mathrm{g}$ GAE/g FW, respectively).

Fresh and frozen dragon fruit samples showed a significant difference in antioxidant activity between the in vitro digestion phases recording the highest DPPH radical inhibition between $27.51 \%$ and $35.59 \%$ during the gastric phase. In contrast, dried dragon fruits showed DPPH inhibition lower than $10 \%$.

This study demonstrated that the polyphenol content and the antioxidant activity of dragon fruits during an in vitro digestion is dependent on the species and the form evaluated. Dragon fruits showed potential to be used in human studies to evaluate their health effects.

\section{Acknowledgments}

To PRODEP-Mexico for supporting this work by the PhD scholarship 511-6/18-7113

\section{References}

1. Coman MM et al. (2018) Eur Food Res Tech 244, 735-745.

2. Lucas-González R et al. (2018) Food Res Int 107, 423-436.

3. Ibrahim SRM et al. (2018) J Food Biochem 42.

4. Choo W-S \& Yong WK (2011) Adv Appl Sci Res 2, 418-425. 